\title{
Genetic risk assessment for cardiovascular disease with seven genes associated with plasma C-reactive protein concentrations in Asian populations
}

\author{
Eun Pyo Hong ${ }^{1}$, Dong Hyun Kim${ }^{2,3}$, Jun Gyo Suh ${ }^{1}$ and Ji Wan Park ${ }^{1}$
}

Plasma C-reactive protein (CRP) level is a predictor of cardiovascular risk. We performed a meta-analysis on the effect of 12 single-nucleotide polymorphisms (SNPs) within 8 candidate loci in 36752 Asians. In addition, we created weighted genetic risk scores (wGRSs) to evaluate the combined effects of genetic variants, which were suggested in the meta-analysis, for predicting the risks of elevated CRP levels as well as increased risks of hypertension and cardiovascular disease (CVD) in 748 Koreans. Nine SNPs located in seven genes, CRP, IL6R, GCKR, IL6, CYP17A1, HNF1A and APOE, were significantly associated with circulating CRP levels in this meta-analysis. Two SNPs, rs7310409 (HNF1A, $\left.P=3.4 \times 10^{-23}\right)$ and rs7553007 $\left(C R P, P=3.4 \times 10^{-17}\right)$, had the most significant effects on CRP levels; and two SNPs, rs2097677 (IL6) and rs1004467 (CYP17A1) have never been found in the previous European meta-analysis. In Koreans, the subjects in the highest wGRS group had an $\sim 2.5$-fold higher mean CRP level compared with those in the lowest wGRS group $\left(P=2.1 \times 10^{-5}\right)$. We observed significant increases in the risks of hypertension (odds ratio $=2.18, P=0.006$ ) and CVD (odds ratio $=9.59, P=3.2 \times 10^{-6}$ ) among the subjects in the highest wGRS group. The wGRS models specific to Koreans may warrant further validation to be used as a proxy for the risk of CVD in Asians. Hypertension Research (2014) 37, 692-698; doi:10.1038/hr.2014.56; published online 27 March 2014

Keywords: cardiovascular disease; C-reactive protein; genetic risk score; meta-analysis

\section{INTRODUCTION}

C-reactive protein (CRP), which is primarily synthesized and secreted in the liver, is present in atherosclerotic lesions but not in normal blood vessel walls. This observation suggests that CRP may play a role in atherogenesis. Thus, the circulating high-sensitivity CRP (hsCRP) concentration is a powerful biomarker that can be used to predict age-related cardioembolic risk. ${ }^{1}$ Previous studies reported that high CRP concentrations in plasma $\left(>3 \mathrm{mgl}^{-1}\right)$ were associated with increased incidence and mortality because of cardiovascular disease (CVD) and metabolic syndromes in elderly populations. ${ }^{2,3}$ Lifestylerelated risk factors, such as cigarette smoking and obesity, that modify the autonomic and inflammatory responses in the vascular system have been shown to be associated with both elevated CRP and the risk of CVD in general populations. ${ }^{4}$

Population-based studies have previously reported statistically significant associations between CRP levels and single-nucleotide polymorphism (SNP) markers located in the following genes: interleukin 6 receptor (IL6R, 1q21); C-reactive protein (CRP, 1q23); glucokinase (hexokinase 4) regulator (GCKR, 2p23); interleukin 6 (IL6, 7p21); cytochrome P450, family 17, subfamily A, polypeptide 1 gene (CYP17A1, 10q24.3); hepatocyte nuclear factor 1 homeobox A (HNF1A, 12q24.2); apolipoprotein C-1 (APOC1, 19q13.2); and apolipoprotein $E$ (APOE, 19q13.2). ${ }^{5-11}$ Liu et al. ${ }^{12}$ found that the CYP17A1 gene variant (rs1004467) was associated with both plasma hsCRP level and risk of hypertension in Chinese patients.

Dehghan et al. ${ }^{13}$ identified 18 loci (for example, CRP, LEPR, GCKR, PABPC4, BCL7B, APOC1, HNF1A and so on) associated with CRP levels in a meta-analysis combining the results of 15 European studies. However, only the HNF1A gene variant has consistently achieved genome-wide significance in four subsequent genome-wide association studies (GWASs) composed of 10112 Japanese patients obtained from Biobank Japan (IL6, HNF1A), ${ }^{14}$ 1709 unrelated Filipino women (CRP, HNF1A), ${ }^{15} \quad 8722$ Koreans $(H N F 1 A)^{16}$ and 6692 Asians living in Singapore (CRP, HNF1A, APOE), respectively. ${ }^{17}$ Among the previous studies published on CRP, eight publications have reported genetic variants associated with CRP concentrations in Asian populations. ${ }^{6,10-12,14-17}$ As far as we know, no meta-analysis of Asian studies has been conducted evaluating the effect of genetic variants on CRP concentrations.

${ }^{1}$ Department of Medical Genetics, Hallym University College of Medicine, Chuncheon, Republic of Korea; ${ }^{2}$ Department of Social and Preventive Medicine, Hallym University College of Medicine, Chuncheon, Republic of Korea and ${ }^{3} \mathrm{Hallym}$ Research Institute of Clinical Epidemiology, Hallym University Sacred Heart Hospital, Anyang, Republic of Korea Correspondence: Dr JW Park, Department of Medical Genetics, Hallym University College of Medicine, 1 Hallymdaehak-gil, Chuncheon, Gangwon-do 200-702, Republic of Korea. 
Initially, we evaluated the effects of 12 SNPs, located in or near 7 candidate genes (that is, IL6R, CRP, GCKR, IL6, CYP17A1, HNF1A and $A P O E$ ), and one intergenic locus (12q23.2), on plasma hsCRP concentration in a community-based cohort study composed of 748 Koreans. Subsequently, we performed a meta-analysis comprising 9 studies involving a total of 36752 Asians to validate the effects of these genetic variants on hsCRP levels. Finally, we developed weighted genetic risk score (wGRS) models that combined the effects of the risk variants to predict high-risk CRP levels as well as increased risks of hypertension and CVD in Koreans.

\section{METHODS}

\section{Genetic association study in a Korean cohort}

Study population and data collection. The population used for the genetic association study consisted of 748 elderly participants with an average age of 68 years. These participants are part of the Hallym Aging Study (HAS), a community-based cohort in Chuncheon city in the Republic of Korea. In 2004, we collected disease status, self-reported demographic and lifestyle data as well as clinical data after informed consent was obtained from each participant at the Hallym University Chuncheon Sacred Heart Hospital. Both systolic blood pressure and diastolic blood pressure were calculated as the averages of three measurements in sitting position. We measured the plasma hsCRP concentrations using a latex-agglutination turbidimetric assay (Pureauto S CRP latex, Daiichi Pure Chemicals, Tokyo, Japan). The plasma concentrations of total cholesterol and high-density lipoprotein cholesterol (HDL-C) were measured by an automatic analyzer (Hitachi 7600-210, Hitachi Medical, Hitachi, Japan). The total cholesterol to HDL-C ratio (T/HDL) was calculated by dividing total cholesterol by HDL-C. The study protocols have been approved by the Institutional Review Board of Hallym University (HIRB-2007-001). Detailed information on the HAS is available elsewhere. ${ }^{18}$

Genotype data analysis. The candidate SNPs for CRP levels were selected by reviewing the literature and public databases, such as dbSNP (www.ncbi. nlm.nih.gov/projects/SNP/), HuGE Navigator (http://hugenavigator.net/ HuGENavigator/home.do) ${ }^{19}$ and SNPinfo (http://snpinfo.niehs.nih.gov/cgibin/snpinfo/snpfunc.cgi). We selected two to five tag SNPs located between $2 \mathrm{~kb} 5^{\prime}$-upstream and $3^{\prime}$-downstream for each of six candidate genes (that is, CRP, IL6R, GCKR, CYP17A1, HNF1A and APOE) and two additional intergenic loci: rs 2097677 , located in the $33.9 \mathrm{~kb} 5^{\prime}$-upstream of the IL6 gene (7p21), and rs10778213, located within a gene desert region (12q23.2). A total of 29 SNPs were genotyped for the 775 Koreans with data on plasma hsCRP levels using the GoldenGate Assay (Illumina, San Diego, CA, USA). Two individuals and four SNPs with genotyping call rates of $<95 \%$ were excluded from subsequent analyses. In addition, we excluded 25 outliers with hsCRP levels $>10 \mathrm{mgl}^{-1}$ that indicates an acute inflammatory disease. We performed preliminary tests to evaluate the Hardy-Weinberg equilibrium, minor allele frequency and pairwise linkage disequilibrium (LD) using Haploview v.4.2. ${ }^{20}$

Because the hsCRP concentrations of the 748 individuals were not normally distributed, the values were transformed into a natural logarithmic scale (ln hsCRP) for subsequent analyses. We performed single marker analyses to test for associations between each of 25 SNPs and $\ln$ hsCRP under an additive model, after adjustments for the six covariates, age, sex, history of hypertension, smoking, body mass index and T/HDL using the STATA software package, v.11.2 (Stata, College Station, TX, USA). Further details can be found elsewhere. $^{21}$

\section{Meta-analysis of Asian populations}

Systematic review of studies. We systematically searched for all published studies of CRP levels in humans, including GWASs, using the Human Genome Epidemiology (HuGE) Literature Finder, a comprehensive search engine of the HuGE Navigator for publications of genetic association studies that is continuously updated from PubMed. ${ }^{19}$ We collected a total of 352 articles, including 12 GWASs, using the keyword 'C-reactive protein' and using study category filtering (that is, 'genetic association' OR 'gene-gene interaction' OR 'gene-environment interaction') and gene filtering for seven candidate genes (that is, 'CRP' OR 'IL6R' OR 'GCKR' OR 'IL6' OR 'CYP17AI' OR 'HNF1A' OR 'APOE'). We excluded animal studies, case-control studies and studies in nonAsian populations including Indo-Europeans. We also excluded studies that did not provide information on their estimated $\beta$-coefficient $(\beta)$ or genotype quality (that is, genotyping success rate, minor allele frequency or HardyWeinberg equilibrium). A total of 8 publications consisting 11 study populations were selected from the literature review (Supplementary Figure S1). The GWAS and the replication of a Japanese study ${ }^{14}$ and three different GWASs by Dorajoo et al. ${ }^{17}$ were analyzed separately in the subsequent analysis. Table 1 provides detailed information regarding the nine Asian studies, including the current study (that is, HAS), analyzed in the current meta-analysis.

Table 1 Characteristics of eligible studies included in the Asian meta-analysis on CRP levels

\begin{tabular}{|c|c|c|c|c|c|c|c|c|}
\hline Cohort study & Population & $\begin{array}{c}\text { Sample } \\
\text { size }\end{array}$ & $\begin{array}{c}\text { Age } \\
\text { years }\end{array}$ & $\begin{array}{c}\text { Female } \\
\%\end{array}$ & $\begin{array}{l}\text { Sample } \\
\text { type }\end{array}$ & Genotyping & $\begin{array}{l}S N P \\
N^{b}\end{array}$ & Adjusted covariates \\
\hline Rhodes et al. ${ }^{6}$ & Filipino & 509 & 38.2 & 79.0 & Serum & MALDI-TOF & 27 & $\begin{array}{l}\text { Age, sex, SLE affection, Apo } \varepsilon 2 \text {, } \\
\text { rs1800796 }\end{array}$ \\
\hline Hsu et al. ${ }^{10}$ & Taiwanese & 617 & 46.0 & 47.0 & Serum & PCR-RFLP & 5 & Age, sex, BMI, smoking \\
\hline Curocihin et al. ${ }^{11}$ & Filipino & 1691 & 21.5 & 47.3 & Plasma & ABI PRISM 7900 & 12 & Sex, pathogenic score, infection status \\
\hline Liu et al. ${ }^{12}$ & Chinese & 3210 & 58.6 & 55.7 & Plasma & GenomeLab SNPstream & 8 & Age, sex, BMI \\
\hline \multirow[t]{2}{*}{ Okada et al. ${ }^{14 \mathrm{c}}$} & Japanese & 10112 & 64.6 & 29.0 & Serum & Illumina HumanHap610-Quad & $\sim 478 \mathrm{~K}$ & Age, sex, BMI, smoking, disease status \\
\hline & & 2742 & 63.1 & 44.2 & Serum & Illumina HumanHap610-Quad & 3 & Age, sex, BMI, smoking, disease status \\
\hline Wu et al. ${ }^{15}$ & Filipino & 1709 & 48.4 & 100.0 & Plasma & Affymetrix SNP Array 5.0 & $\sim 2.07 \mathrm{M}$ & Age, household assets, income and so on \\
\hline Kong et al. ${ }^{16}$ & Korean & 8722 & 52.2 & 52.7 & Serum & Affymetrix SNP Array 5.0 & $\sim 1.35 \mathrm{M}$ & Age, sex, BMI and smoking \\
\hline \multirow[t]{3}{*}{ Dorajoo et al. ${ }^{17}$} & Chinese & 2179 & 48.0 & 53.4 & Serum & $\begin{array}{l}\text { Illumina 1Mduo., HumanHap } \\
610 Q u a d\end{array}$ & $\sim 1.75 \mathrm{M}$ & Age, sex, population stratification \\
\hline & Malay & 2275 & 59.1 & 50.5 & Serum & HumanHap 610Quad & $\sim 1.56 \mathrm{M}$ & Age, sex, population stratification \\
\hline & Asian-Indian & 2238 & 58.0 & 48.9 & Serum & HumanHap 610Quad & $\sim 1.53 \mathrm{M}$ & Age, sex, population stratification \\
\hline HAS & Korean & 748 & 68.0 & 56.2 & Plasma & Illumina Golden Gate Assay & 25 & $\begin{array}{l}\text { Age, sex, BMI, smoking, hypertension, } \\
\text { T/HDL }\end{array}$ \\
\hline
\end{tabular}

Abbreviations: BMI, body mass index; CRP, C-reactive protein; HAS, Hallym Aging Study; MALDI-TOF, matrix-assisted laser desorption and ionization-time of flight; PCR-RFLP, PCR with restriction enzyme digestion; SLE, systemic lupus erythematosus; SNP, single-nucleotide polymorphism; T/HDL, total cholesterol to high-density lipoprotein cholesterol ratio.

CRP concentrations $\left(\mathrm{mgl}^{-1}\right)$ were log-transformed in all studies.

a Mean age of each study population.

bNumber of single-nucleotide polymorphisms under study.

'A total of 10112 Japanese analyzed for $~ 478000$ SNPs in the genome-wide association study and 2742 Japanese analyzed for the association of 3 SNPs identified in the initial study. 
SNP selection. We initially selected 51 SNP markers from 7 candidate genes and 1 intergenic SNP, rs10778213, in this review. We evaluated a pairwise LD of these SNPs using the LD TAG SNP Selection (TagSNP) of the SNPinfo Web Server that interfaces with dbSNP (http://snpinfo.niehs.nih.gov/snpinfo/ snptag.htm) and excluded SNPs in complete LD with each other $\left(r^{2}=1\right)$ because they provide redundant information. We analyzed a total of 12 SNPs that were studied in at least 2 of 11 studies included in the current metaanalysis.

Statistical analysis. We combined the genotype data for 748 Koreans and 36004 Asians. Using the genome-wide association meta-analysis (GWAMA) software package developed by Magi and Morris ${ }^{22}$ (http://www.well.ox.ac.uk/ gwama/), we estimated the combined $\beta$ s and $P$-values under both fixed- and random-effect inverse variance models with an assumption of an additive genetic model for the $12 \mathrm{SNPs}$. We performed the Cochran $Q$ test using the $I^{2}$ statistic to evaluate heterogeneity across studies. For each SNP, we summarized the $\beta$ with $95 \%$ confidence interval for each study and for the combined study in a forest plot. We generated funnel plots for detecting publication bias and heterogeneity across studies. To correct for multiple testing of the 12 SNP markers, we applied a significance threshold of $P<0.004$ based on a Bonferroni correction.

\section{Functional analysis in silico}

A total of nine SNP markers were evaluated to predict their biological functions, such as nonsynonymous SNPs, stop codons, transcription factor binding sites, splicing regulators and miRNA binding sites, in Asian populations using the online SNP Function Prediction (FuncPred) tool (http:// snpinfo.niehs.nih.gov/snpinfo/snpfunc.htm). We further evaluated the functional impact of five nonsynonymous SNPs (that is, rs2228145, rs1260326, rs1169288, rs2464196 and rs429358) using the web-based program PolyPhen-2 (http://genetics.bwh.harvard.edu/pph2).

\section{Genetic risk assessments for high hsCRP level, hypertension and CVD in Koreans}

We calculated wGRS by multiplying the number of risk alleles $(0,1$ and 2$)$ for each SNP by the exponentiated $\beta$ coefficent for that SNP. Although only three SNPs remained statistically significant after Bonferroni correction in the HAS, eight SNPs achieved the Bonferroni-adjusted significance level in the current meta-analysis, and the $\mathrm{rs} 1004467(P=0.017)$ is a well-known risk variant for hypertension in Asian populations. ${ }^{12}$ Thus, we summed the wGRS across nine SNPs (that is, rs2228145, rs7553007, rs1260326, rs2097677, rs1004467, rs1169288, rs7310409, rs2464196 and rs429358), categorized it into five groups and evaluated association of wGRS with hsCRP levels using a generalized linear model adjusted for the six covariates listed above. We further compared the wGRS under the assumption of an additive genetic model (that is, additive-wGRS) with the wGRS that combined the effect of each SNP estimated under the best-fitting genetic model (additive, dominant or recessive) (that is, model-specific wGRS) for all three phenotypes (Table 3 and Supplementary Table S4).

To assess genetic risks of hypertension and CVD, we created wGRS models composed of four CRP-associated SNPs (that is, rs2228145, rs2097677, rs1004467 and rs7310409) that were also associated with either hypertension or CVD. Hypertensive cases included 455 participants with systolic blood pressure/diastolic blood pressure of $\geq 140 / 90 \mathrm{~mm} \mathrm{Hg}$ or having a history of hypertension. Cases of CVD were defined as 110 participants who had a history of CHD or stroke. We selected a common control group of 101 subjects with systolic blood pressure $<120 \mathrm{~mm} \mathrm{Hg}$, diastolic blood pressure $<80 \mathrm{~mm}$ $\mathrm{Hg}$ and no history of hypertension and CVD. We summed the wGRS across four SNPs and categorized the wGRS into three groups. We performed multiple logistic regression analyses between the wGRS and either hypertension or CVD, after adjusting for age, sex, smoking, drinking and body mass. Furthermore, we compared this model-specific wGRS with the wGRS after adjustment for the covariates specific to hsCRP (that is, age, sex, smoking, body mass index and T/HDL).

\section{RESULTS}

Our meta-analysis consisted of 36752 individuals obtained from 11 Asian study populations and a Korean cohort. The two Japanese studies by Okada et al. ${ }^{14}$ consisted of a GWAS and a replication study obtained from the BioBank Japan Project. The two Filipino studies were obtained from the same cohort, the Cebu Longitudinal Health and Nutrition Survey (CLHNS). ${ }^{11,15}$ Dorajoo et al. ${ }^{17}$ performed three independent GWASs of Chinese, Malay and Asian-Indian populations. Table 1 summarizes the population characteristics and study designs of the 12 studies. We found significant difference across studies; age, gender ratio and other covariate adjustment in each study. The mean age of subjects in most of the studies was $>45$ years, except for the two Filipino studies (mean age was 21.5 and 38.2 years, respectively). ${ }^{6,11}$ The gender ratio was 29 to $79 \%$ women, with Wu et al. ${ }^{15}$ including only women. Nine studies, including the Korean cohort, recruited subjects from the general population; ${ }^{10-12,15-17}$ however, three studies consisted of patients only. ${ }^{6,14}$ Eight studies, including the Korean cohort, excluded outliers with CRP levels $>10 \mathrm{mgl}^{-1}$ before analysis; ${ }^{6,11,15-17}$ however, no exclusion criteria for CRP level were observed in the other four studies. ${ }^{10,12,14}$ All studies log-transformed the CRP levels because of the highly skewed distribution before analysis. Most studies adjusted for age, sex and additional covariates in their statistical analyses, although the identity of these covariates was not identical between studies.

We summarized the results of the previous association studies for 12 SNPs on CRP levels using an additive model (Supplementary Table S1). The results of the generalized linear model for 12 SNPs, after adjustments for 6 covariates, using an additive model in 748 Koreans are presented in Table 2. Seven SNPs exhibited significant associations with plasma CRP levels, and three of these SNPs, rs2228145 (IL6R), rs7310409 (HNF1A) and rs429358 (APOE), remained significant following a Bonferroni correction $(P<0.004)$. The Asian meta-analysis, including the Korean population, identified 9 out of 12 SNPs that were significantly associated with CRP levels under the additive model. Eight SNPs in the meta-analysis were significant following a Bonferroni correction. Two SNPs, rs7310409 (HNF1A, 12q24.2) and rs429358 (APOE, 19q13.2), exhibited the lowest $P$-value $\left(\beta=0.06, P=3.4 \times 10^{-23}\right)$ and the highest effect size $\left(\beta=0.27, P=7.1 \times 10^{-8}\right)$, respectively. Six SNPs revealed statistically significant heterogeneity $(P<0.05)$. Therefore, we performed a subgroup analysis and these SNPs demonstrated strong evidence for an association with CRP levels in the homogenous subgroup analysis (that is, rs2228145, $P=6.7 \times 10^{-8}$; rs7553007, $P=3.4 \times 10^{-17}$; rs2097677, $P=2.5 \times 10^{-11}$; rs1169288, $P=3.9 \times 10^{-4}$; rs7310409, $P=3.4 \times 10^{-23} ;$ rs2464196, $\left.P=3.6 \times 10^{-12}\right)$. Two SNPs, rs2097677 (IL6) and rs1004467 (CYP17A1), that were insignificant in the Korean study became statistically significant in the meta-analysis, but not rs1004467, after Bonferroni correction. We presented both forest plots and funnel plots of the rs7310409 (Figures 1a and 1b) and the other eight SNPs (Supplementary Figures S2-S9). Genetic heterogeneity and/or publication bias was suspected in the two Filipino studies (Figure 1b). ${ }^{11,15}$

We identified possible biological functions for six out of the nine SNP markers implicated by the Asian meta-analysis as being associated with CRP levels. The rs2228145 (Asp358Ala) SNP, which is located in exon 9 of the IL6R gene, is an alternative exonic splicing enhancer that regulates the expression of IL6R. The rs 1260326 (Leu446Pro, GCKR) was predicted to be potentially damaging to the base sequence. Three other nonsynonymous SNPs, rs1169288 (Ile27Leu, HNF1A), rs2464196 (Ser487Asn, HNF1A) and rs429358 


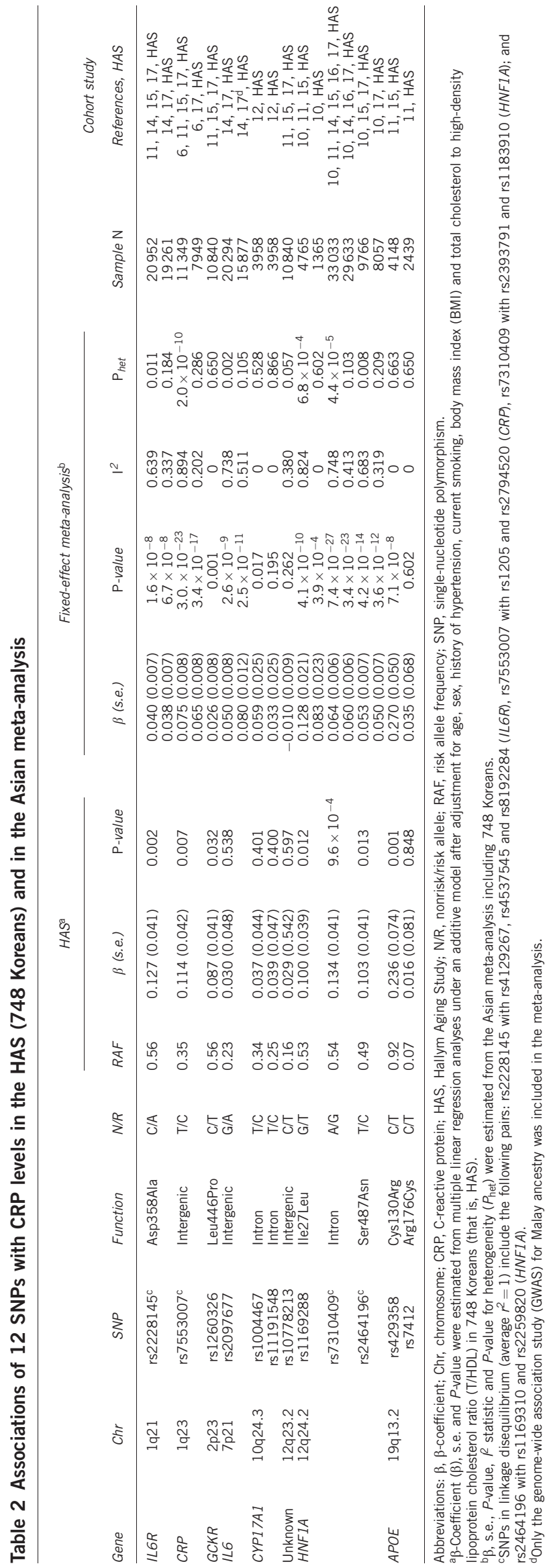

(Cys130Arg, APOE), result in amino acid substitutions. An intronic SNP rs1004467 (CYP17A1) is located at a microRNA-binding site (Supplementary Table S2).

We tested for the combined effect of the nine SNPs (Figure 2a) using a wGRS model with adjustments for six covariates on hsCRP level in 748 Koreans (Figure 2b). Initially, we evaluated the combined effect of seven SNPs that showed significant associations in the Korean study; however, the mean hsCRP concentration of the highest additive-wGRS group calculated using seven SNPs (11.01-14.85) was lower $\left(1.77 \mathrm{mgl}^{-1}\right)$ than that of the highest additive-wGRS group calculated using the nine SNPs suggested in the Asian meta-analysis (15.01-17.94, $\left.2.55 \mathrm{mgl}^{-1}\right)$. As expected, the hsCRP concentration linearly increased with increasing wGRS in Koreans. The group of subjects with the highest additive-wGRS had an $\sim 2.5$-fold higher mean CRP level compared with the subjects with the lowest additive-wGRS (3.39-6.00, $1.03 \mathrm{mg} \mathrm{l}^{-1}$; Figure 2). The additive-wGRS model appears to fit the CRP data better than the model-specific wGRS (Table 3 and Supplementary Table S3).

Three SNPs, rs2228145 (IL6R), rs2097677 (IL6) and rs7310409 (HNF1A), out of 12 SNPs were associated with CVD, whereas two SNPs, rs1004467 and rs11191548 (CYP17A1), were associated with hypertension at $P<0.05$ (Supplementary Table S3 and Figure 3a). The inclusion of SNPs not being associated with the disease weakened the predictability of wGRS model. The wGRS composed of 4 SNPs associated with either hypertension or CVD have higher predictability than the wGRS of 9 SNPs selected for CRP (that is, $57.9 \%$ vs. $56.1 \%$ for hypertension; $65.7 \%$ vs. $65.1 \%$ for CVD, data not shown). Thus, we showed the combined effects of four SNPs in the wGRS models for two diseases (Figure 3a). The model-specific wGRS fits the data better than the additive-wGRS model for both hypertension and CVD (Table 3 and Supplementary Table S4). Subjects in the highest wGRS group (10.01-12.52) had a 2.1-fold and a 9.6-fold increased risks of hypertension $(P=0.008)$ and $\operatorname{CVD}\left(P=3.2 \times 10^{-6}\right)$, respectively, compared with those in the lowest wGRS group (1.45-8.00; Figure $3 \mathrm{~b}$ and Table 3). Associations between the wGRS models and either hypertension or CVD were significantly determined by different sets of covariates; and the risk of disease significantly increased along with the increase in both wGRS models (Table 3 and Supplementary Table S5).

\section{DISCUSSION}

We evaluated the effects of 12 SNPs of 7 candidate genes and 1 intergenic locus previously indicated as being associated with CRP levels in a large-scale meta-analysis of 36752 Asians. We showed that the alleles of SNPs from five genes, IL6R, CRP, GCKR, HNF1A and $A P O E$, increased the CRP levels in both Asian and European populations, even though the minor allele was not always identical between ethnicities. ${ }^{6-11,13-17}$ A previous European meta-analysis on CRP levels did not report two genetic variants, CYP17A1 (rs1004467) and IL6 (rs2097677). ${ }^{13}$ Although the minor C allele of rs10778213, which is located within a gene desert region (12q23.2), was strongly associated with low CRP levels in 6345 white women from the Women's Genome Health Study $\left(P=1.2 \times 10^{-10}\right)$, it was not associated with CRP levels in Asian populations. ${ }^{7,11,15-17}$ Individuals with high wGRS appeared to be at considerably higher risk than those with any one risk allele. The combined effect of four SNPs, rs2228145 (IL6R), rs2097677 (IL6), rs7310409 (HNF1A) and the rs1004467 (CYP17A1), highly increased the risk of both hypertension and CVD in the current study.

Both IL6R and CRP gene polymorphisms were shown to be strongly associated with CRP levels through a pathway associated 
a

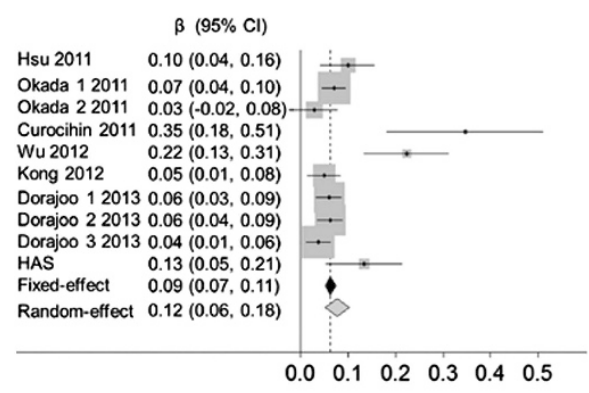

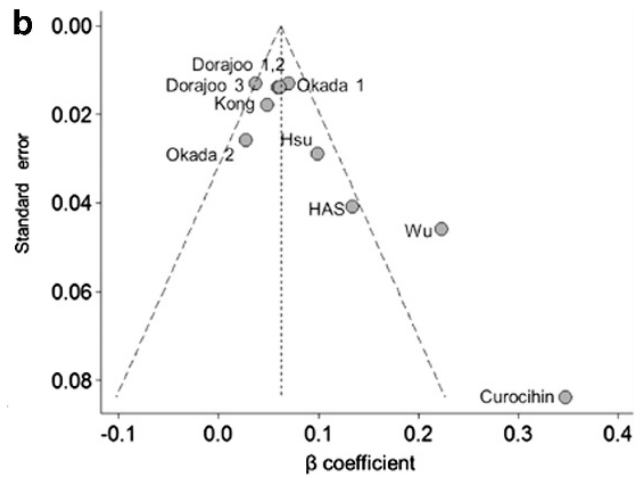

Figure 1 Forest (a) and funnel (b) plots for rs7310409 (HNF1A) in the Asian meta-analysis using an inverse variance model. The first author and the Korean cohort (Hallym Aging Study (HAS)) is represented in (a) and (b). Okada 1 and Okada 2 by Okada et al. ${ }^{14}$ show the results of a genome-wide association study (GWAS) and a replication study, respectively. Dorajoo 1-3 by Dorajoo et al. ${ }^{17}$ show the results of 2179 Chinese, 2275 Malay and 2238 Asian-Indian GWASs, respectively. The horizontal line indicates the $95 \%$ confidence interval $(\mathrm{Cl})$; the shaded square indicates the weight of each study. The estimates of effect size from the fixed-effect (black diamond) or random-effect (gray diamond) inverse variance models are shown in (a). The divergent lines are the pseudo $95 \% \mathrm{Cls}$ and the central line indicates the pooled $\beta$ in (b).

a
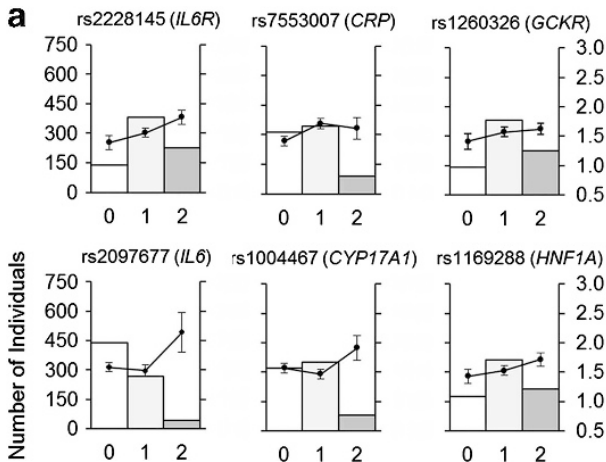

rs1169288 (HNF1A) rs7310409(HNF1A)

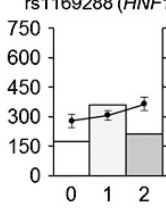

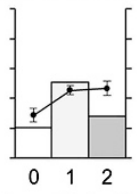

Number of risk alleles
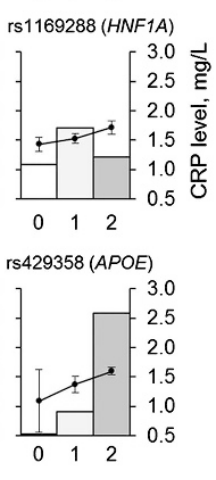

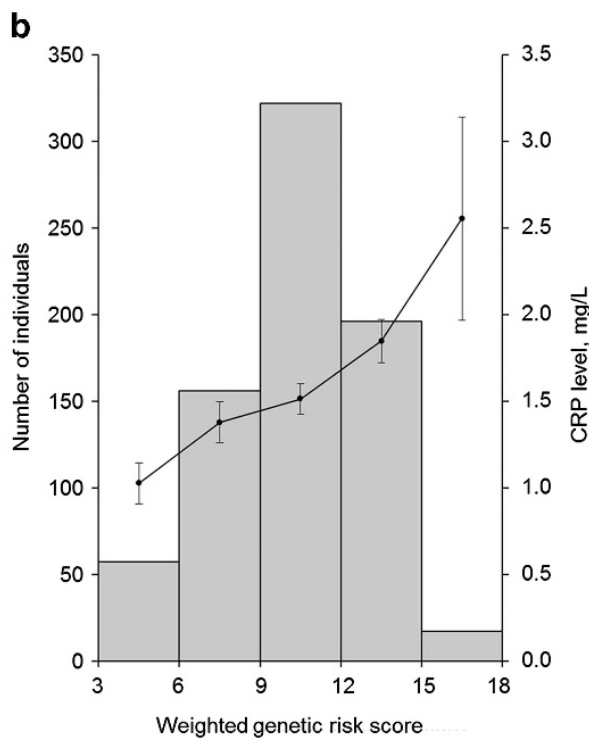

Figure 2 Mean C-reactive protein (CRP) concentrations according to the number of risk allele (0,1 and 2) for nine single-nucleotide polymorphisms (SNPs) (a) and the weighted genetic risk score (wGRS) for the combined effects of those nine SNPs (b). Bars indicate the number of individuals by risk allele score (left vertical axis). Dots connected by a solid line indicate the mean CRP levels according to each risk allele score and a short dash represents the standard error of the mean CRP levels (right vertical axis). In (b), the X axis is categorized by the range of wGRSs: 3.39-6.00, 6.01-9.00, 9.01-12.00, 12.0115.00 and $15.01-17.94$.

with the innate immune response. ${ }^{13,23}$ The 358Ala allele of rs2228145 (in strong LD with rs4129267 and rs4537545) located in the IL6R gene was significantly associated with reduced CRP levels and reduced risk of $\mathrm{CHD}$ in two recent studies; ${ }^{24,25}$ the protective effect was consistently observed in the current study (odds ratio $=0.32$, $P=0.01$ ). The SNP rs1205 (in complete LD with rs7553007), located within the $3^{\prime}$ flanking sequence of the CRP gene, was associated with regulating CRP expression. However, none of the CRP gene variants showed a consistent association with CHD risk in both the European study ${ }^{24}$ and the current Korean study. The rs1004467 (CYP17A1), which exhibited a suggestive association with increased CRP levels in our meta-analysis $(P=0.017)$, was associated with blood pressure and hypertension in 3210 unrelated elderly Chinese Hans. ${ }^{12}$ To date, there is no published report on a potential relationship between the gene variants of IL6 and HNF1A and CVD.
Although the IL6 gene variant (rs2097677) has not been identified in either European GWAS $^{7,8}$ or meta-analysis, ${ }^{13}$ a Japanese study suggested its association with CRP levels. ${ }^{14}$ Common variations of GCKR, HNF1A and APOE genes are related to abnormal metabolism and metabolic pathway regulation. ${ }^{7}$ An activating glucokinase mutation regulated by the GCKR gene causes maturity onset diabetes of the young type 2 (MODY2) and leads to persistent hyperinsulinemic hypoglycemia. In addition, the Leu446 allele of the GCKR coding variant (rs1260326) is associated with increased triglycerides and CRP levels, but lower fasting glucose, in the general population. ${ }^{26}$ The HNF1A gene consists of 10 exons spanning an $\sim 23-\mathrm{kb}$ region and encodes a transcription factor regulating the expressions of liver-related genes, including CRP gene that is associated with CRP level, lipid profiles, glucose metabolism and inflammation response. This gene variant therefore plays a crucial 
Table 3 Associations of model-specific weighted genetic risk score groups with hsCRP level, hypertension and CVD in 748 Koreans

\begin{tabular}{|c|c|c|c|}
\hline wGRS group & Subjects, N (\%) & $\beta / O R(95 \% C l)^{b}$ & P-value \\
\hline \multicolumn{4}{|l|}{ hsCRP } \\
\hline $3.39-6.00$ & $97(7.6)$ & Reference & \\
\hline $6.01-9.00$ & 249 (20.9) & $0.10(-0.08$ to 0.28$)$ & 0.279 \\
\hline $9.01-12.00$ & $371(43.0)$ & $0.34(0.18$ to 0.51$)$ & $6.3 \times 10^{-5}$ \\
\hline $12.01-15.00$ & $29(26.2)$ & 0.57 (0.27 to 0.88$)$ & $2.3 \times 10^{-4}$ \\
\hline $15.01-17.94$ & $2(2.3)$ & 1.17 (0.15 to 2.19 ) & 0.024 \\
\hline \multicolumn{4}{|l|}{ Hypertension } \\
\hline $1.45-8.00$ & $128(28.1) / 44(43.6)$ & Reference & \\
\hline $8.01-10.00$ & $147(32.3) / 25(24.7)$ & 1.89 (1.04 to 3.42 ) & 0.037 \\
\hline $10.01-12.52$ & $180(39.6) / 32(31.7)$ & 2.13 (1.22 to3.72) & 0.008 \\
\hline \multicolumn{4}{|l|}{$C V D$} \\
\hline $1.45-8.00$ & $21(18.9) / 44(43.6)$ & Reference & \\
\hline $8.01-10.00$ & $29(26.1) / 25(24.7)$ & 4.66 (1.80 to12.03) & 0.002 \\
\hline $10.01-12.52$ & $61(54.0) / 32(31.7)$ & 9.59 (3.70 to 24.85$)$ & $3.2 \times 10^{-6}$ \\
\hline \multicolumn{4}{|c|}{$\begin{array}{l}\text { Abbreviations: } \beta, \beta \text {-coefficient; } \mathrm{Cl} \text {, confidence interval; CVD, cardiovascular disease; hsCRP, } \\
\text { high-sensitivity C-reactive protein; OR, odds ratio; wGRS, weighted genetic risk score. } \\
\text { aSubjects denote total number of subjects for hsCRP and numbers of cases and controls for } \\
\text { hypertension and CVD. } \\
\text { b } \beta, 95 \% \mathrm{Cl} \text { and } P \text {-values were estimated for the combined effect of nine single-nucleotide } \\
\text { polymorphisms (SNPs) on hsCRP from a generalized linear model adjusted for age, sex, history } \\
\text { of hypertension, cigarette smoking, body mass index (BMI) and total cholesterol to high-density } \\
\text { lipoprotein cholesterol ratio (T/HDL); OR, } 95 \% \mathrm{Cl} \text { and } P \text {-values were estimated for the } \\
\text { combined effect of four SNPs on hypertension and CVD from logistic regression models } \\
\text { adjusted for age, sex, cigarette smoking, alcohol drinking and BMI under the best-fitting } \\
\text { genetic model (that is, additive, dominant and recessive). }\end{array}$} \\
\hline
\end{tabular}

role in controlling CRP level and developing $\mathrm{CHD} .{ }^{27}$ Rare mutations of HNF1A polymorphisms cause MODY3 and are associated with the metabolic pathway for insulin secretion. ${ }^{28}$ APOE polymorphisms are associated with cholesterol release from blood vessels and the antiinflammatory system. ${ }^{29}$ The rs429358 (Cys130Arg) variant of the $A P O E$ gene is also known to increase the risk of Alzheimer disease in multiethnic groups. ${ }^{30}$

We observed considerable differences not only in age, gender ratio and covariate adjustment, but also in the allele frequencies of SNPs among studies of five different Asian populations: Japanese, Korean, Chinese, Malay, Asian-Indian, Filipino and Taiwanese. However, the associations of the six SNPs; rs2228145, rs7553007, rs2097677, rs1169288, rs7310409, and rs2464196 that revealed the presence of a substantial heterogeneity, remained significant in homogenous subgroup analyses.

In conclusion, we highlight the major functions of the genetic variants that are crucial to immune response and abnormal metabolic pathways related to chronic inflammation. These findings warrant further investigation to validate the effects of SNPs associated with elevated CRP levels on the increased risk for developing CVD in a large-scale Asian population-based study. If the wGRS models developed in the current study are validated, they can serve as proxy measures for the increased genetic risk of hypertension and atherosclerotic CVD. a
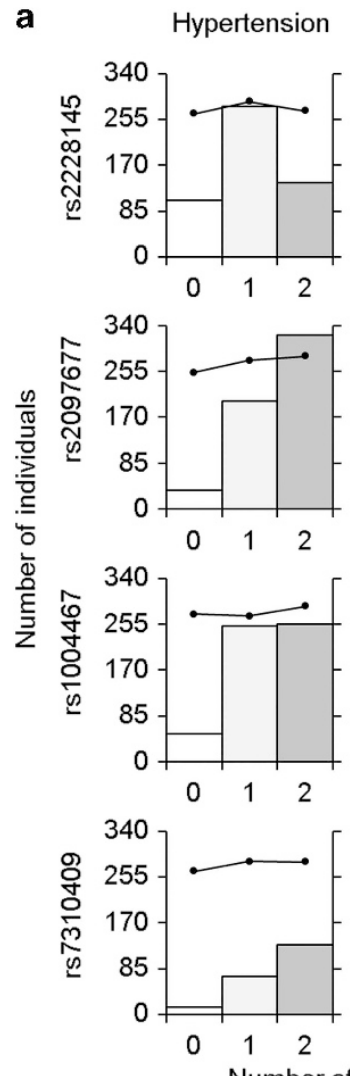

CVD

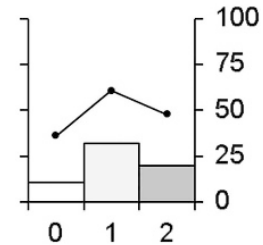

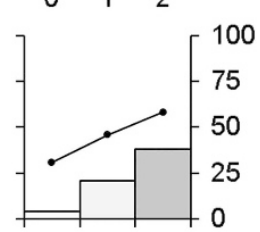
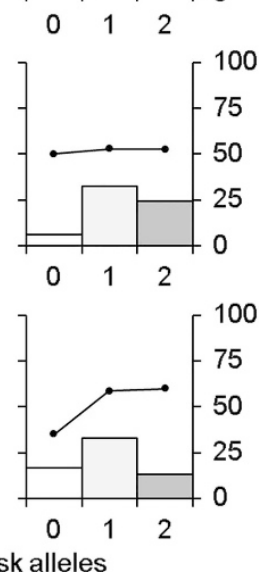

b

Hypertension

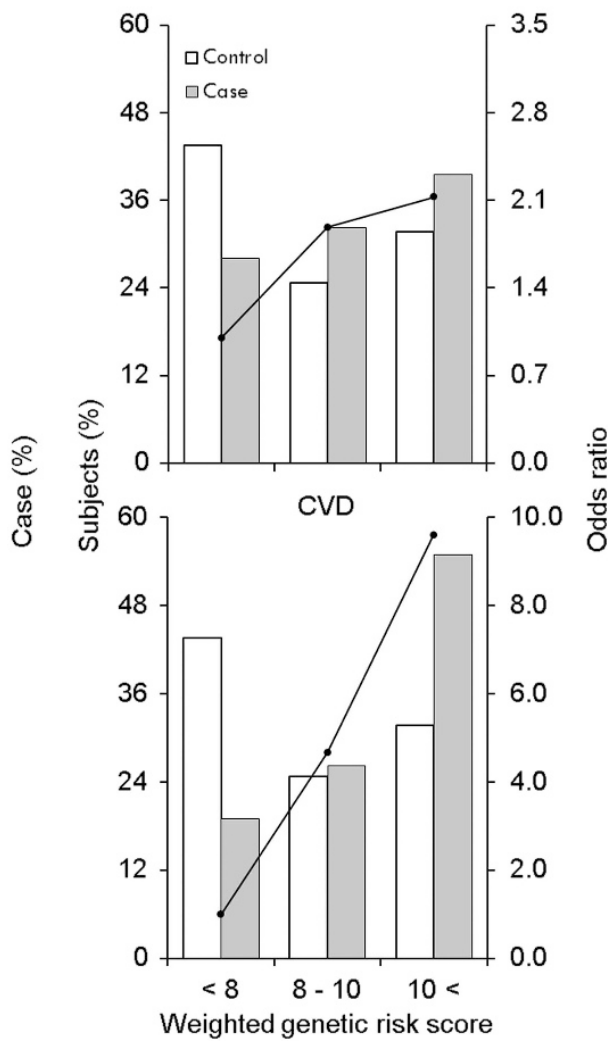

Figure 3 Proportion of cases with hypertension or coronary heart disease (CVD) according to the number of risk allele (0, 1 and 2$)$ for four single-nucleotide polymorphisms (SNPs) (a) and the weighted genetic risk score (wGRS) for the combined effects of those four SNPs (b). Bars indicate the number of individuals by risk allele score (left vertical axis). Dots connected by a solid line indicate the proportion of cases according to each risk allele score (right vertical axis). In (b), the $X$ axis is categorized by the range of wGRS: 1.45-8.00, 8.01-10.00 and 10.01-12.52. Gray bars and white bars indicate the percentage of case subjects and control subjects in the range of wGRS. Dots connected by a solid line indicate odds ratios (ORs) by wGRS groups. 


\section{CONFLICT OF INTEREST}

The authors declare no conflict of interest.

\section{ACKNOWLEDGEMENTS}

This research was supported by the Basic Science Research Program through the National Research Foundation of Korea (NRF) funded by the Ministry of Education, Science and Technology, Republic of Korea (2009-0090837) and Hallym University Research Fund, 2013 (HRF-201301-013). We thank Dr Lange LA who provided the allele frequencies of 1691 Filipino females obtained from the CLHNS cohort.

1 Verma S, Szmitko PE, Ridker PM. C-reactive protein comes of age. Nat Clin Pract Cardiovasc Med 2005; 2: 29-36.

2 Ridker PM. Role of inflammatory biomarkers in prediction of coronary heart disease. Lancet 2001; 358: 946-948.

3 Choi EY, Park EH, Cheong YS, Rheem I, Park SG, Yoo S. Association of C-reactive protein with the metabolic risk factors among young and middle-aged Koreans. Metabolism 2006; 55: 415-421.

4 Madsen C, Nafstad P, Eikvar L, Schwarze PE, Rønningen KS, Haaheim LL. Association between tobacco smoke exposure and levels of C-reactive protein in the Oslo II Study. Eur J Epidemiol 2007; 22: 311-317.

5 Shen J, Ordovas JM. Impact of genetic and environmental factors on hsCRP concentrations and response to therapeutic agents. Clin Chem 2009; 55: 256-264.

6 Rhodes B, Wong A, Navarra SV, Villamin C, Vyse TJ. Genetic determinants of basa C-reactive protein expression in Filipino systemic lupus erythematosus families. Genes Immun 2008; 9: 153-160.

7 Ridker PM, Pare G, Parker A, Zee RY, Danik JS, Buring JE, Kwiatkowski D, Cook NR, Miletich JP, Chasman DI. Loci related to metabolic-syndrome pathways including LEPR, HNF1A, IL6R, and GCKR associate with plasma C-reactive protein: the Women's Genome Health Study. Am J Hum Genet 2008; 82: 1185-1192.

8 Reiner AP, Barber MJ, Guan Y, Ridker PM, Lange LA, Chasman DI, Walston JD, Cooper GM, Jenny NS, Rieder MJ, Durda JP, Smith JD, Novembre J, Tracy RP, Rotter JI, Stephens M, Nickerson DA, Krauss RM. Polymorphisms of the HNF1A gene encoding hepatocyte nuclear factor- 1 alpha are associated with C-reactive protein. Am J Hum Genet 2008; 82: 1193-1201.

9 Hubacek JA, Peasey A, Pikhart H, Stavek P, Kubinova R, Marmot M, Bobak M. APOE polymorphism and its effect on plasma C-reactive protein levels in a large general population sample. Hum Immunol 2010; 71: 304-308.

$10 \mathrm{Hsu}$ LA, Ko YL, Teng MS, Wu S, Chou HH, Chang PY, Chang $\mathrm{HH}$. Effect of obesity on the association between common variations in the HNF1A gene region and C-reactive protein level in Taiwanese. Clin Chim Acta 2011; 412: 725-729.

11 Curocichin G, Wu Y, McDade TW, Kuzawa CW, Borja JB, Qin L, Lange EM, Adair LS, Lange LA, Mohlke KL. Single-nucleotide polymorphisms at five loci are associated with C-reactive protein levels in a cohort of Filipino young adults. J Hum Genet 2011; 56: 823-827.

12 Liu C, Li H, Qi Q, Lu L, Gan W, Loos RJ, Lin X. Common variants in or near FGF5, CYP17A1 and MTHFR genes are associated with blood pressure and hypertension in Chinese Hans. J Hypertens 2011; 29: 70-75.

13 Dehghan A, Dupuis J, Barbalic M, Bis JC, Eiriksdottir G, Lu C, Pellikka N, Wallaschofski H, Kettunen J, Henneman P, Baumert J, Strachan DP, Fuchsberger C Vitart V, Wilson JF, Paré G, Naitza S, Rudock ME, Surakka I, de Geus EJ, Alizadeh BZ, Guralnik J, Shuldiner A, Tanaka T, Zee RY, Schnabel RB, Nambi V, Kavousi M, Ripatti S, Nauck M, Smith NL, Smith AV, Sundvall J, Scheet P, Liu Y, Ruokonen A, Rose LM, Larson MG, Hoogeveen RC, Freimer NB, Teumer A, Tracy RP, Launer LJ, Buring JE, Yamamoto JF, Folsom AR, Sijbrands EJ, Pankow J, Elliott P, Keaney JF, Sun W Sarin AP, Fontes JD, Badola S, Astor BC, Hofman A, Pouta A, Werdan K, Greiser KH, Kuss O, Meyer zu Schwabedissen HE, Thiery J, Jamshidi Y, Nolte IM, Soranzo N, Spector TD, Völzke H, Parker AN, Aspelund T, Bates D, Young L, Tsui K, Siscovick DS, Guo X, Rotter JI, Uda M, Schlessinger D, Rudan I, Hicks AA, Penninx BW, Thorand B, Gieger C, Coresh J, Willemsen G, Harris TB, Uitterlinden AG, Järvelin MR, Rice K, Radke D, Salomaa V, Willems van Dijk K, Boerwinkle E, Vasan RS, Ferrucci L, Gibson QD, Bandinelli S, Snieder H, Boomsma DI, Xiao X, Campbell H, Hayward C, Pramstaller PP, van Duijn CM, Peltonen L, Psaty BM, Gudnason V, Ridker PM, Homuth G, Koenig W, Ballantyne CM, Witteman JC, Benjamin EJ, Perola M, Chasman DI. Meta-analysis of genome-wide association studies in $>80000$ subjects identifies multiple loci for C-reactive protein levels. Circulation 2011; 123: 731-738.

14 Okada Y, Takahashi A, Ohmiya H, Kumasaka N, Kamatani Y, Hosono N, Tsunoda T, Matsuda K, Tanaka T, Kubo M, Nakamura Y, Yamamoto K, Kamatani N. Genome-wide association study for C-reactive protein levels identified pleiotropic associations in the IL6 locus. Hum Mol Genet 2011; 20: 1224-1231.
15 Wu Y, McDade TW, Kuzawa CW, Borja J, Li Y, Adair LS, Mohlke KL, Lange LA. Genomewide association with C-reactive protein levels in CLHNS: evidence for the CRP and HNF1A loci and their interaction with exposure to a pathogenic environment. Inflammation 2012; 35: 574-583.

16 Kong M, Lee C. Genetic associations with C-reactive protein level and white blood cel count in the KARE study. Int J Immunogenet 2013; 40: 120-125.

17 Dorajoo R, Li R, Ikram MK, Liu J, Froguel P, Lee J, Sim X, Ong RT, Tay WT, Peng C, Young TL, Blakemore Al, Cheng CY, Aung T, Mitchell P, Wang JJ, Klaver CC, Boerwinkle E, Klein R, Siscovick DS, Jensen RA, Gudnason V, Smith AV, Teo YY, Wong TY, Tai ES, Heng CK, Friedlander Y. Are C-reactive protein associated genetic variants associated with serum levels and retinal markers of microvascular pathology in Asian populations from Singapore? PLoS One 2013; 8: e67650.

18 Cho YG, Song HJ, Lee SK, Jang SN, Jeong JY, Choi YH, Hong KS, Choi MG, Kang SH, Kang JH, Kim DH, Caterson I. The relationship between body fat mass and erectile dysfunction in Korean men: Hallym Aging Study. Int J Impot Res 2009; 21: 179-186.

19 Yu W, Gwinn M, Clyne M, Yesupriya A, Khoury MJ. A navigator for human genome epidemiology. Nat Genet 2008; 40: 124-125.

20 Barrett JC, Fry B, Maller J, Daly MJ. Haploview: analysis and visualization of LD and haplotype maps. Bioinformatics 2005; 21: 263-265.

21 Hong EP, Kim DH, Suh JG, Park JW. Analyses of longitudinal effects of geneenvironment interactions on plasma C-reactive protein levels: the Hallym Aging Study. Genes Genom 2013; 35: 131-139.

22 Magi R, Morris AP. GWAMA: software for genome-wide association meta-analysis. BMC Bioinformatics 2010; 11: 288.

23 Reich D, Patterson N, Ramesh V, De Jager PL, McDonald GJ, Tandon A, Choy E, Hu D, Tamraz B, Pawlikowska L, Wassel-Fyr C, Huntsman S, Waliszewska A, Rossin E, Li R, Garcia M, Reiner A, Ferrell R, Cummings S, Kwok PY, Harris T, Zmuda JM, Ziv EHealth, Aging and Body Composition (Health $\mathrm{ABC}$ ) Study. Admixture mapping of an allele affecting interleukin 6 soluble receptor and interleukin 6 levels. Am J Hum Genet 2007; 80: 716-726.

24 Elliott $P$, Chambers JC, Zhang W, Clarke R, Hopewell JC, Peden JF, Erdmann J Braund P, Engert JC, Bennett D, Coin L, Ashby D, Tzoulaki I, Brown IJ, Mt-Isa S, McCarthy MI, Peltonen L, Freimer NB, Farrall M, Ruokonen A, Hamsten A, Lim N, Froguel P, Waterworth DM, Vollenweider P, Waeber G, Jarvelin MR, Mooser V, Scott J, Hall AS, Schunkert H, Anand SS, Collins R, Samani NJ, Watkins H, Kooner JS. Genetic loci associated with $\mathrm{C}$-reactive protein levels and risk of coronary heart disease. JAMA 2009; 302: 37-48.

25 IL6R Genetics Consortium Emerging Risk Factors Collaboration, Sarwar N, Butterworth AS, Freitag DF, Gregson J, Willeit P, Gorman DN, Gao P, Saleheen D, Rendon A, Nelson CP, Braund PS, Hall AS, Chasman DI, Tybjærg-Hansen A Chambers JC, Benjamin EJ, Franks PW, Clarke R, Wilde AA, Trip MD, Steri M, Witteman JC, Qi L, van der Schoot CE, de Faire U, Erdmann J, Stringham HM, Koenig W, Rader DJ, Melzer D, Reich D, Psaty BM, Kleber ME, Panagiotakos DB, Willeit J, Wennberg P, Woodward M, Adamovic S, Rimm EB, Meade TW, Gillum RF, Shaffer JA, Hofman A, Onat A, Sundström J, Wassertheil-Smoller S, Mellström D, Gallacher J, Cushman M, Tracy RP, Kauhanen J, Karlsson M, Salonen JT, Wilhelmsen L, Amouyel P, Cantin B, Best LG, Ben-Shlomo Y, Manson JE, Davey-Smith G, de Bakker PI, O'Donnell CJ, Wilson JF, Wilson AG, Assimes TL, Jansson JO, Ohlsson C, Tivesten $\AA$, Ljunggren Ö, Reilly MP, Hamsten A, Ingelsson E, Cambien F, Hung J, Thomas GN, Boehnke M, Schunkert H, Asselbergs FW, Kastelein JJ, Gudnason V, Salomaa V Harris TB, Kooner JS, Allin KH, Nordestgaard BG, Hopewell JC, Goodall AH, Ridker PM, Hólm $H$, Watkins $H$, Ouwehand WH, Samani NJ, Kaptoge S, Di Angelantonio E, Harari O, Danesh J. Interleukin-6 receptor pathways in coronary heart disease: a collaborative meta-analysis of 82 studies. Lancet 2012; 379: 1205-1213.

26 Orho-Melander M, Melander O, Guiducci C, Perez-Martinez P, Corella D, Roos C, Tewhey R, Rieder MJ, Hall J, Abecasis G, Tai ES, Welch C, Arnett DK, Lyssenko V, Lindholm E, Saxena R, de Bakker PI, Burtt N, Voight BF, Hirschhorn JN, Tucker KL, Hedner T, Tuomi T, Isomaa B, Eriksson KF, Taskinen MR, Wahlstrand B, Hughes TE, Parnell LD, Lai CQ, Berglund G, Peltonen L, Vartiainen E, Jousilahti P, Havulinna AS, Salomaa V, Nilsson P, Groop L, Altshuler D, Ordovas JM, Kathiresan S. Common missense variant in the glucokinase regulatory protein gene is associated with increased plasma triglyceride and C-reactive protein but lower fasting glucose concentrations. Diabetes 2008; 57: 3112-3121.

27 Armendariz AD, Krauss RM. Hepatic nuclear factor 1-alpha: inflammation, genetics, and atherosclerosis. Curr Opin Lipidol 2009; 20: 106-111.

28 Yamagata K, Oda N, Kaisaki PJ, Menzel S, Furuta H, Vaxillaire M, Southam L, Cox RD, Lathrop GM, Boriraj VV, Chen X, Cox NJ, Oda Y, Yano H, Le Beau MM, Yamada S, Nishigori H, Takeda J, Fajans SS, Hattersley AT, Iwasaki N, Hansen T, Pedersen O, Polonsky KS, Bell GI. Mutations in the hepatocyte nuclear factor-1alpha gene in maturity-onset diabetes of the young (MODY3). Nature 1996; 384: 455-458.

29 Mazzone T, Reardon C. Expression of heterologous human apolipoprotein E by $\mathrm{J774}$ macrophages enhances cholesterol efflux to HDL3. J Lipid Res 1994; 35: 1345-1353.

30 Tang MX, Maestre G, Tsai WY, Liu XH, Feng L, Chung WY, Chun M, Schofield P, Stern Y, Tycko B, Mayeux R. Relative risk of Alzheimer disease and age-at-onset distributions, based on APOE genotypes among elderly African Americans, Caucasians, and Hispanics in New York City. Am J Hum Genet 1996; 58: 574-584.

Supplementary Information accompanies the paper on Hypertension Research website (http://www.nature.com/hr) 